\title{
S1 Leitlinie zur Diagnostik und Therapie der kutanen Larva migrans (Creeping Disease)
}

\section{Zusammenfassung}

Die kutane Larva migrans ist eine in ihrem klinischen Bild typische Hautinfektion, die durch aktives Eindringen und anschließende epidermale Wanderung von Nematodenlarven hervorgerufen wird. Dieses typische klinische Bild wird durch Larven von Hakenwürmern, meist Ancylostoma braziliense, selten andere bei Kaniden und Feliden vorkommende Hakenwurmarten, verursacht.

Ziele der Leitlinie sind die Verbesserung der Versorgung der Patienten durch Optimierung von Diagnostik und Therapie bei Infektionen mit Larva migrans cutanea sowie die Verbesserung der Kenntnisse von Ärztinnen und Ärzte über aktuelle Therapieoptionen.

Schlüsselwörter: Leitlinie, Diagnostik, Therapie, kutane Larva migrans, Creeping Disease

\author{
Cord Sunderkötter ${ }^{1}$ \\ Esther von Stebut ${ }^{2}$ \\ Helmut Schöfer ${ }^{3}$ \\ Martin Mempel ${ }^{4}$ \\ Dieter Reinel $^{5}$ \\ Gerd Wolf ${ }^{6}$ \\ Volker Meyer ${ }^{1}$ \\ Alexander $\mathrm{Nast}^{7}$ \\ Gerd-Dieter Burchard ${ }^{8,9}$
}

1 Klinik für Hautkrankheiten allgemeine Dermatologie und Venerologie,

Universitätsklinikum Münster (UKM), Münster, Deutschland

2 Hautklinik der Universitätsmedizin, Johannes-GutenbergUniversität, Mainz, Deutschland

3 Klinik für Dermatologie, Venerologie und Allergologie, Klinikum der Johann Wolfgang Goethe Universität, Frankfurt/M., Deutschland

4 Elmshorn, Deutschland

5 Hamburg, Deutschland

6 Grafschaft-Ringen, Deutschland

7 Division of Evidence based Medicine (dEBM), Klinik für Dermatologie, Venerologie und Allergologie, Charité Universitätsmedizin Berlin, Deutschland

8 Sektion Tropenmedizin und Infektiologie,

Unversitätsklinikum Hamburg-Eppendorf, Hamburg, Deutschland

9 ifi - Institut für Interdisziplinäre Medizin, Hamburg, Deutschland 


\section{Ziele der Leitlinie}

Verbesserung der Versorgung der Patienten durch Optimierung von Diagnostik und Therapie bei Infektionen mit Larva migrans cutanea. Verbesserung der Kenntnisse von Ärztinnen und Ärzten über aktuelle Therapieoptionen.

\section{Methodik}

S1 Leitlinie, nicht systematische Literaturrecherche, Konsensusprozess im Umlaufverfahren

\section{Einleitung}

Die kutane Larva migrans ist eine in ihrem klinischen Bild typische Hautinfektion, die durch aktives Eindringen und anschließende epidermale Wanderung von Nematodenlarven hervorgerufen wird. Dieses typische klinische Bild wird durch Larven von Hakenwürmern, (meist Ancylostoma braziliense, selten andere bei Kaniden und Feliden vorkommende Hakenwurmarten, siehe unten) verursacht. Die Weiterentwicklung dieser tierischen Hakenwurmlarven ist im Menschen nicht möglich.

Abzugrenzen ist die Larva migrans cutanea von der Larva currens. Hierunter versteht man eine Reaktion auf Larven von Strongyloides stercoralis, vorwiegend am Rumpf oder Gesäß lokalisiert. Diese zeigt eine weniger irreguläre Wanderung, in weiten und weniger scharf begrenzten Gängen die Extremitäten entlang (bis zu $5 \mathrm{~cm}$ pro Stunde - daher Larva currens) und verschwindet meist rasch. Auf die Larva currens wird in dieser Leitlinie nicht eingegangen. Eine Larva migrans cutanea (als Syndrom) ist ebenfalls abzugrenzen von dem Symptom „Creeping eruption“ - hierunter versteht man wandernde subkutane Schwellungen mit Rötung und Urticaria der darüberliegenden Haut, zum Beispiel bei Gnathostomiasis, kutaner Paragonimiasis (Lungenegel-Infektion) oder Fasziolose (Leberegel-Infektion), ähnliche wandernde Symptome sind auch möglich bei einer Myiasis („migratory myiasis“), also bei einem Befall mit Fliegenmaden.

\section{Erreger}

Bei der kutanen Larva migrans durch tierpathogene Nematodenlarven (meist Ancylostoma braziliense, aber auch Ancylostoma caninum oder Uncinaria stenocephala) bleibt die Infektion auf die Haut beschränkt. Die Larven dringen in die intakte Epidermis (Füße, Gesäß oder andere exponierte Areale) ein, vermutlich über Haarfollikel oder Schweißdrüsen, und bohren sich unter Hinterlassung charakteristisch gewundener Gänge durch die Epidermis. Die Basalmembran vermögen sie nicht zu durchdringen. Sie bewegen sich je nach Art unterschiedlich schnell vorwärts, in der Regel bis zu $1 \mathrm{~cm}$ pro Tag. Die juckenden Effloreszenzen sind Ausdruck einer Immunreaktion auf die Wurmlarven und ihre Produkte. Für diese auf Hunde und Katzen spezialisierten Parasiten stellt der Mensch einen Fehlwirt dar, und die Larven sterben nach einigen Wochen ab.

\section{Epidemiologie}

Die kutane Larva migrans ist verbreitet an warmen, sandigen Orten (vor allem Strände) in Südamerika, Afrika, in der Karibik, Südostasien, südöstliche Staaten der USA, teilweise auch am Mittelmeer [1].

Die Prävalenz ist dort hoch, wo in Gegenden mit warmfeuchtem Klima barfuß laufende Menschen mit Exkrementen freilaufender Tiere in Kontakt kommen. In Deutschland tritt die Erkrankung vor allem auf bei Reisenden, die Strände mit frei laufenden infestierten Tieren besucht haben. Auswertungen in internationalen Beobachtungsnetzwerken zu Erkrankungen bei Reisenden und Migranten zeigen, dass die kutane Larva migrans eine der am häufigsten importierten Hautkrankheiten ist. So machte unter innen in einer weltweiten Untersuchung die Larva migrans cutanea $10 \%$ der dermatologischen Diagnosen aus [2]. Auch in Auswertungen einzelner Zentren ist die Larva migrans cutanea eine der häufigsten Diagnosen (in Frankreich [3], oder in Spanien [4]).

\section{Klinik der Larva migrans}

Einige Tage nach Kontakt mit verseuchten Böden entsteht zunächst eine Papel, von dieser breitet sich jeweils ein gewundener Gang mit lokal begrenzter Entzündungsreaktion aus (Abbildung 1). Die Inkubationszeit kann Tage, aber auch Wochen oder Monate betragen [5]. Infestationen mit mehreren Larvae migrantes gleichzeitig sind möglich (Abbildung 2). Die Gänge können mit einer serösen Flüssigkeit gefüllt sein und es können sich als Folge der Entzündungsreaktion Bläschen bilden. Juckreiz ist ein typisches Symptom. Er kann bereits vor Auftreten der ersten Effloreszenz beginnen und nimmt im Verlauf deutlich zu [6]. Die kutane Form ist selbstlimitierend, da die tierpathogenen Larven im Menschen absterben. In einigen Fällen können die Effloreszenzen über mehrere Monate persistieren, ohne dass histologisch Larven detektiert werden; die Ursache ist nicht ganz geklärt und kann u.a. dennoch in einer Persistenz einer Larve oder in einer anhaltenden Immunreaktion auf Larvenbestandteile liegen [7]. Aufgrund der Eintrittspforte oder aufgrund von Kratzexkoriationen kann sich jedoch sekundär eine bakterielle Infektion (zum Beispiel ein Erysipel) entwickeln, eine vor allem in den Endemiegebieten häufige Komplikation. 


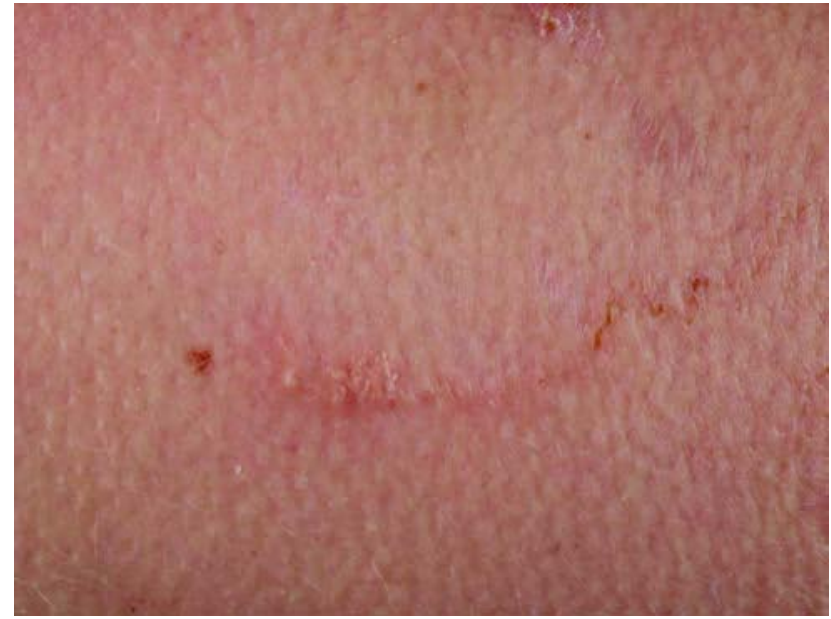

Abbildung 1: Serpiginöse Spur der kutanen Larva migrans mit nur mäßiger Entzündungsreaktion

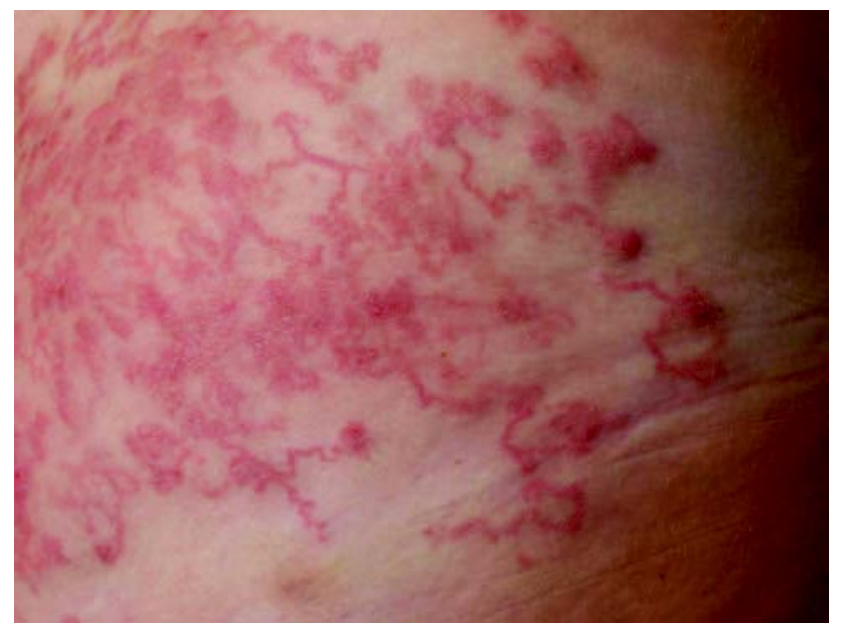

Abbildung 2: Befall mit mehreren Nematoden und Begleitentzündungsreaktion

\section{Prophylaxe}

Auf möglicherweise befallenen Stränden und Böden sollte Schuhwerk mit festen Sohlen (zum Beispiel Sandalen) getragen werden. Badegäste sollten sich nicht direkt auf den Sand und auch nicht auf Handtücher legen, sondern Matratzen oder Liegen nutzen. Strandareale, die regelmäßig von den Gezeiten gespült werden, sollten bevorzugt werden. Wenn möglich, sollten Strände genutzt werden, an denen sich weder Katzen noch Hunde aufhalten.

\section{Diagnostik/Differentialdiagnose}

Die serpiginösen Exantheme sind pathognomonisch, die entsprechende Reise- und Expositionsanamnese untermauert den Verdacht.

Bei der Infestation mit Strongyloides stercoralis beginnen die Effloreszenzen meist in der Perinealregion und breiten sich in weiten und weniger scharf begrenzten Gängen an den Extremitäten aus (bis zu $5 \mathrm{~cm}$ pro Stunde, daher Larva currens genannt).

\section{Indikation zur Therapie}

Eine Infektion mit einer kutanen Larva migrans ist selbstlimitierend und endet in der Regel nach 1-3 Monaten mit dem Tod und der Resorption der Larve. Eine Therapie sollte vor allem wegen des ausgeprägten und anhaltenden Juckreizes, der psychischen Belastung eines Parasitenbefalls und der Gefahr einer möglichen Superinfektion zum Beispiel nach Kratzexkoriationen erfolgen.

\section{Therapie}

Lange wurde in Deutschland und anderen Ländern die Lokaltherapie mit Thiabendazol in einer lipophilen Grundlage empfohlen [8]. Hierzu gab es zwar kaum Studien, aber der Therapie wurde eine hohe therapeutische Breite zugeschrieben. Thiabendazol ist seit 1988 nicht mehr in Deutschland zugelassen und auch nicht mehr als Reinsubstanz in Pharmaqualität erhältlich.

Wir unterbreiten nach Durchsicht der Literatur und nach Erfahrungsberichten folgende Vorschläge zur Therapie:

\section{Erwachsene und Jugendliche:}

1. Ivermectin 1x $200 \mu \mathrm{g} / \mathrm{kgKG}$ (Stromectol $3 \mathrm{mg}$, Mectizan $3 \mathrm{mg}$, Ivermec $6 \mathrm{mg}$, Revectina $6 \mathrm{mg}$ Tablette) Orale Gabe; 2 Stunden vor und nach Einnahme des Medikamentes sollte keine Nahrung aufgenommen werden. In der Regel reicht die einmalige Gabe aus, wenn nach 10 Tagen aber keine Besserung eingetreten ist, kann eine zweite Dosis gegeben werden [9]. Falls bei ausgeprägtem Befall Ivermectin nicht verfügbar, nicht wirksam oder nicht verträglich ist, stellt die systemische Therapie mit Albendazol eine Alternative dar.

2. Albendazol $800 \mathrm{mg} /$ Tag oral für 3 Tage (zum Beispiel Eskazole 400 mg Tabletten); bei Jugendlichen unter 60 kg, aber über 6 Jahre: 15 mg/kg Körpergewicht bis maximal $800 \mathrm{mg} / \mathrm{Tag}$ ), bei Unverträglichkeit (gastrointestinale Beschwerden) Dosisreduktion auf $400 \mathrm{mg} /$ Tag für 5 Tage.

Einnahme in 2 Einzeldosen morgens und abends zu den Mahlzeiten.

3. Lokaltherapie mit Albendazol $10 \%$ in lipophiler Grundlage (zum Beispiel Albendazol 1200 mg (entsprechend 3 Tabletten zu $400 \mathrm{mg}$ ) in Vaselinum album ad 12,0) drei mal täglich für 7-10 Tage auf einem genügend großen Areal auftragen. Anwendung vor allem bei Kindern unter 15 kg Körpergewicht (kein Ivermectin) oder Kindern unter 6 Jahren (keine genügende Erfahrung mit Albendazol) sowie bei Patienten mit diskretem Befund (nur eine Läsion, kein quälender Juckreiz) und bei Patienten, die eine Systemtherapie ablehnen. Siehe auch Kommentar unten zum Bezug von Albendazol für die Lokaltherapie).

Begleitend empfehlen wir zur Milderung der Entzündung und des Juckreizes eine kurzzeitige (1-5 Tage) 
Lokaltherapie mit einer glukokortikoidhaltigen Creme (Expertenmeinung) und bei Bedarf systemische Anthistaminika zur Juckreizstillung.

Schwangerschaft und Stillzeit:

In der Schwangerschaft dürfen weder Ivermectin noch Albendazol eingenommen werden. Hier können wir bislang nur die oben erwähnte symptomatische Therapie empfehlen, das heißt zur Milderung der Entzündung und des Juckreizes eine Lokaltherapie mit einer glukokortikoidhaltigen Creme und bei Bedarf die bei Schwangerschaft zugelassenen systemischen Anthistaminika zur Stillung des Juckreizes.

Unter einer wirksamen Behandlung sollte der Juckreiz nach 3, die Dermatitis nach 7 Tagen deutlich rückläufig sein.

\section{Nicht empfohlen werden:}

1. Topische Anwendung von Mebendazol

Begründungen: a) Die Wirkung scheint im Vergleich zu Albendazol bei Larva migrans und allgemein gegen Hakenwürmer schwächer zu sein [10], [11]; b) die intestinale Resorption ist nicht ausreichend, so dass es unter den gebräuchlichen Dosierungen zwar gegen intestinale Nematoden, nicht aber gegen Nematoden im Gewebe wirksam ist; c) entsprechend waren keine Publikationen zum systemischen Einsatz von Mebendazol gegen Larva migrans auffindbar.

2. Kryotherapie (obwohl mitunter genannt), da der Aufenthaltsort der Larve (meist 1-2 cm im Umkreis des sichtbaren Gangendes) nicht genau bekannt ist [12] und ihre Empfindlichkeit auf Kälte in der Haut nicht hoch ist ( $>5$ min Überleben bei $-21^{\circ} \mathrm{C}$ ).

\section{Kommentar zu Ivermectin}

Ivermectin ist in Deutschland nur als Tierarzneimittel unter dem Namen Eraquell Tabs 20 mg ad usum veterinarium (zum tierärztlichen Gebrauch) zugelassen. In Frankreich, Belgien und den USA besteht eine Zulassung am Menschen für die Behandlung einer viszeralen Form der verwandten intestinalen Strongyloidiasis durch den Zwergfadenwurm Strongyloides stercoralis.

Die Tabletten lassen sich aber über jede Apotheke aus dem Ausland unter den Handelsnamen Stromectol ${ }^{\circledR} 3 \mathrm{mg}$, Ivermec ${ }^{\circledR} 6$ mg oder Revectina ${ }^{\circledR} 6$ mg Tabl. (N1) beziehen. Der Patient soll darüber aufgeklärt werden, dass Ivermectin in Deutschland nicht und im Ausland nicht direkt für Larva migrans Infektionen zugelassen ist. Die Aufklärung sollte zusammen mit einer Nutzen-Risiko-Abwägung des Off-Label Einsatzes in der Patientenakte dokumentiert werden.

Bei Kindern unter 15 kg Körpergewicht und bei Schwangeren ist die Anwendung kontraindiziert. Beeinträchtigende unerwünschte Wirkungen sind transient und zudem unter der millionenfachen Anwendung gegen Onchozerkose selten beschrieben worden [13].

\section{Kommentar zu Albendazol systemisch}

Albendazol ist für die systemische Gabe zur Zeit nur in einer Packungsgröße mit 60 Tabletten (N3) zu einem Verkaufspreis von 590,38€ [Importe: 549,09-556,09€/ Preise: Stand: 05/2013] als Eskazole ${ }^{\circledR}$ [14]) erhältlich. Zu wünschen wäre eine kleinere Packungsgröße, da die Inzidenz der Fälle einer Larva migrans nicht niedrig ist und somit häufiger nur kleine Mengen erforderlich sind. Wenn Eskazole ${ }^{\circledR}$ verordnet wird, sind die Apotheken auch bei Verwendung von nur 6 Tabletten für die orale Therapie laut der rechtsverbindlichen „Hilfstaxe für Apotheken“ berechtigt, auf den Einkaufspreis der Packung 90\% aufzuschlagen und die ganze Packung zu berechnen. Klinikapotheken können für den stationären Gebrauch in der internen Verrechnung nur die benutzte Menge in Rechnung stellen. Das wäre theoretisch auch für Apotheken möglich, steht aber nicht im Einklang mit der „Hilfstaxe für Apotheken“. Albendazol ist in Deutschland als Antihelminikum zugelassen, darunter unter anderem für den Behandlungsversuch bei Befall mit Strongyloides stercoralis (siehe oben), aber nicht für die Behandlung von Larva migrans, so dass sein Gebrauch hierfür außerhalb der Zulassung liegt (so genannter „Off Label“ Einsatz). Folglich soll der Patient darüber informiert werden, und die Aufklärung sollte zusammen mit einer Nutzen-Risiko-Abwägung des Off-Label Einsatzes in der Patientenakte dokumentiert werden.

\section{Kommentar Albendazol topisch}

Die vorgeschlagene Rezeptur entstammt einem Fallbericht [15]: Albendazol 1,2 g, Vaselinum album ad 12,0 g. Die Koautorin nutzt Albendazol 10,0, Paraff. subliq. 10,0, weißes Vaselin ad 100,0.

Wenn für die Rezeptur Eskazole ${ }^{\circledR}$ verordnet wird, sind die Apotheken auch bei Verwendung von nur 3 Tabletten berechtigt, den vollen Preis zu erheben (siehe oben).

Seit 2013 wird Albendazol aber auch in Form der Reinsubstanz für Apotheken angeboten (von der Firma Fagron, Barsbüttel) und könnte dadurch kostengünstiger für eine lokale Anwendung verarbeitet werden. Unsere bisherige Erfahrung hat indes gezeigt, dass Lieferschwierigkeiten auftreten können (offenbar vor allem aufgrund mangelnder Reinheit der von unterschiedlichen Zulieferern stammenden Substanzen). Außerdem müssen Apotheken nach Auslieferung einer reinen Substanz mit Analysenzertifikat vor einer pharmazeutischen Verarbeitung von Albendazol einen Identitätsnachweis mittels Infrarotspektroskopie und Dünnschichtchromatographie durchführen. Über Infrarotspektroskope verfügen aber nur wenige Apotheken.

Daher sollte sich der verordnende Arzt vorher erkundigen a) welche Apotheke den geforderten Identitätsnachweis durchzuführen vermag, und b) ob für diese Apotheke Albendazol lieferbar ist. Für die Apotheke ist beachtenswert, dass sie für die Rezeptur eventuell eine größere Mindestmenge abnehmen und bezahlen muss als sie für ein einzelnes Rezept berechnen darf. 
Sollten diese Erfordernisse aber erfüllt sein, ergäbe sich daraus für den Patienten eine deutlich kostengünstigere Lokalbehandlung als unter Verwendung von Eskazole ${ }^{\circledR}$. Daher empfehlen wir, dass solche Einrichtungen, die häufiger Patienten mit Larva migrans behandeln, sich langfristig mit einer Apotheke über die oben genannten Bedingungen verständigen, damit sie nicht jedes Mal erneut abgeklärt werden müssen.

\section{Beurteilung der Wirksamkeit}

Es gibt nur wenige vergleichende prospektive randomisierte Studien. Die einmalige Gabe von Ivermectin ( $n=10$ Patienten) zeigte sich in einer vergleichenden randomisierten Studie wirksamer als $400 \mathrm{mg}$ Albendazol ( $n=11$ Patienten) [16]. Für Ivermectin im Vergleich zu Albendazol könnte auch sprechen, dass es bei Strongyloidiasis (also einer Erkrankung durch einen verwandten Wurm) in einer prospektiven Studie in einer Einmaldosierung wirksamer war als $800 \mathrm{mg}$ Albendazol für 7 Tage [17].

Neben vergleichenden Studien gibt es eine Reihe von teilweise retrospektiven Auswertungen mit einzelnen Medikamenten:

Ivermectin: In zwei kleineren Studien in den 90iger Jahren war eine Einmaldosis bei Patienten aus Kamerun und bei französischen Touristen gut wirksam [18], [19]. Dies ließ sich dann in größeren Studien bestätigen [9]. In einer Studie in Paris wurden 62 Europäer mit Ivermectin behandelt, nach einer Dosis betrug die Heilungsrate 77\%, mit ein bis zwei zusätzlichen Dosen 97\% [20]. Schließlich gibt es noch eine weitere aktuelle Studie aus Frankreich, in welcher 59 von 62 Patienten mit Ivermectin geheilt werden konnten [21] und eine Studie aus einem brasilianischen Endemiegebiet der zufolge alle 92, zum Teil schwer befallenen Patienten 2-4 Wochen nach einmaliger Gabe von 200 g/kg signifikant angesprochen haben [22].

Albendazol: In einer retrospektiven Erhebung an 78 Patienten mit kutaner Larva migrans führten 1x400 mg/Tag Albendazol p.o. nach 7 Tagen bei allen zur Heilung. Zwei Patienten hatten unter der Medikation Übelkeit oder verstärkten Pruritus [23]. In weiteren publizierten Fallserien oder retrospektiven Erhebungen kamen verschiedene Dosen und Behandlungsdauern zum Einsatz, wobei unter 800 mg/Tag für 3 Tage 100\%ige Heilungsraten, unter $400 \mathrm{mg} / \mathrm{Tag}$ hingegen einzelne Therapieversager berichtet wurden [24], [25]. In der Dosis von 800 mg/Tag scheint es gegenüber Ivermectin eine höhere Rate an unerwünschten (meist gastrointestinalen) Nebenwirkungen zu haben [26].

Zur Lokaltherapie mit Albendazol wurden ein Fallbericht [15] und eine Empfehlung im Rahmen einer Übersicht [11] gefunden. Topisches Albendazol hat in dem Fallbericht bei 2 Kindern 1 Woche nach Behandlung zu einer rezidivfreien Abheilung geführt [15]. Außer diesem Fallbericht sind keine genügenden Daten zu Hautpenetration, Wirksamkeit und Verträglichkeit bekannt. Die Autorinnen und Autoren dieser Leitlinien haben einzelne Fälle eben- falls erfolgreich topisch mit 10\% Albendazol behandelt. Die Empfehlung, Albendazol 10\% in lipophiler Grundlage, 3-mal täglich für 7-10 Tage auf einem genügend großen Areal aufzutragen, entstand in Analogie zur früher oft verwandten Thiabendazol-haltigen Rezeptur und gemäß des publizierten Fallberichtes. Die Größe des Behandlungsareals ergibt sich aufgrund des Wissens, dass die Larve oft mehrere Zentimeter weiter gewandert ist, als die Entzündung anzeigt.

Glukokortikoide: Direkte Evidenz zur Wirksamkeit einer begleitenden Lokaltherapie mit Glukokortikoiden zur Milderung der Entzündung und des Juckreizes bei Larva migrans liegen nicht vor.

\section{Anmerkungen}

\section{Gültigkeit}

Die vorliegende Leitlinie hat eine Gültigkeit bis April 2017. Die Autorengruppe entscheidet über die Notwendigkeit einer Aktualisierung. Erstellungsdatum: Mai 2013.

\section{ICD-Code (ICD 10)}

B76.9

\section{AWMF-Register-Nummer}

$013 / 087$

\section{Interessenkonflikte}

Siehe Anhang 1.

\section{Finanzierung}

Die Erstellung der Leitlinie erfolgte als Eigenleistung der Autoren. Es erfolgte keinerlei Finanzierung und es erfolgte keine äußere Einflussnahme auf die Inhalte der Leitlinie.

\section{Genehmigungen}

Autorisiert durch Deutsche Dermatologische Gesellschaft (DDG).

Autorisiert für die elektronische Publikation: AWMF online. Autorisiert für Publikation in GMS ID durch John Wiley \& Sons Ltd Blackwell Verlag im Rahmen der interdisziplinären Erstellung dieser Leitlinie unter Mitwirkung der PEG (ursprünglich erschienen im Journal der Deutschen Dermatologischen Gesellschaft 2014 [27]).

\section{Beteiligte Fachgesellschaften und deren Vertreter}

- Deutsche Dermatologische Gesellschaft (DDG)//Arbeitsgemeinschaft für dermatologische Infektiologie und Tropendermatologie (ADI-TD): E. von Stebut-Bor- 
schitz, H. Schöfer, M. Mempel, D. Reinel, V. Meyer, A. Nast, C. Sunderkötter

- Deutsche Gesellschaft für Tropenmedizin und Internationale Gesundheit e.V. (DTG): G.-D. Burchard, D. Reinel

- Society for Dermatology in the Tropics: D. Reinel

- Paul Ehrlich Gesellschaft: C. Sunderkötter

\section{Anhänge}

Verfügbar unter

http://www.egms.de/en/journals/id/2014-2/id000011.shtml

1. Anhang1_id000011.pdf (122 KB)

Interessenkonflikte

\section{Literatur}

1. Galanti B, Fusco FM, Nardiello S. Outbreak of cutaneous larva migrans in Naples, southern Italy. Trans R Soc Trop Med Hyg. 2002 Sep-Oct;96(5):491-2. DOI: 10.1016/S00359203(02)90415-3

2. Lederman ER, Weld LH, Elyazar IR, von Sonnenburg F, Loutan L, Schwartz E, Keystone JS; GeoSentinel Surveillance Network. Dermatologic conditions of the ill returned traveler: an analysis from the GeoSentinel Surveillance Network. Int J Infect Dis. 2008 Nov;12(6):593-602. DOI: 10.1016/j.ijid.2007.12.008

3. Caumes E, Carrière J, Guermonprez G, Bricaire F, Danis M, Gentilini M. Dermatoses associated with travel to tropical countries: a prospective study of the diagnosis and management of 269 patients presenting to a tropical disease unit. Clin Infect Dis. 1995 Mar;20(3):542-8. DOI: 10.1093/clinids/20.3.542

4. Ramírez-Olivencia G, Bru Gorraiz FJ, Rivas González P, Lago Núñez M, Herrero Mendoza MD, Puente Puente S. Patologia dermatologica y medicina tropical. Resultados de un estudio prospectivo (2004-2007)[Skin diseases and tropical medicine. Results from a prospective study (2004-2007)]. Rev Clin Esp. 2009 Dec;209(11):527-35. DOl: 10.1016/S00142565(09)73058-9

5. Siriez JY, Angoulvant F, Buffet P, Cleophax C, Bourrat E. Individual variability of the cutaneous larva migrans (CLM) incubation period. Pediatr Dermatol. 2010 Mar-Apr;27(2):211-2. DOI: 10.1111/j.1525-1470.2010.01107.x

6. Feldmeier $\mathrm{H}$, Schuster A. Mini review: Hookworm-related cutaneous larva migrans. Eur J Clin Microbiol Infect Dis. 2012 Jun;31(6):915-8. DOI: 10.1007/s10096-011-1404-x

7. Veraldi S, Persico MC, Francia C, Schianchi R. Chronic hookwormrelated cutaneous larva migrans. Int J Infect Dis. 2013 Apr;17(4):e277-9. DOI: 10.1016/j.ijid.2012.11.002

8. Hochedez P, Caumes E. Hookworm-related cutaneous larva migrans. J Travel Med. 2007 Sep-Oct;14(5):326-33. DOI: 10.1111/j.1708-8305.2007.00148.x

9. Van den Enden E, Stevens A, Van Gompel A. Treatment of cutaneous larva migrans. N Engl J Med. 1998 Oct 22;339(17):1246-7. DOI: 10.1056/NEJM199810223391714

10. Steinmann P, Utzinger J, Du ZW, Jiang JY, Chen JX, Hattendorf J, Zhou $\mathrm{H}$, Zhou XN. Efficacy of single-dose and triple-dose albendazole and mebendazole against soil-transmitted helminths and Taenia spp.: a randomized controlled trial. PLoS ONE. 2011;6(9):e25003. DOI: 10.1371/journal.pone.0025003

11. Wolf P, Ochsendorf FR, Milbradt R. Aktuelle Therapiemöglichkeiten bei Larva migrans cutanea [Current therapeutic possibilities in cutaneous larva migrans]. Hautarzt. $1993 \mathrm{Jul} ; 44(7): 462-5$.
12. Albanese G, Venturi C, Galbiati G. Treatment of larva migrans cutanea (creeping eruption): a comparison between albendazole and traditional therapy. Int J Dermatol. 2001 Jan;40(1):67-71. DOI: 10.1046/j.1365-4362.2001.01103.x

13. Meinking TL, Taplin D, Hermida JL, Pardo R, Kerdel FA. The treatment of scabies with ivermectin. N Engl J Med. 1995 Jul;333(1):26-30. DOI: 10.1056/NEJM199507063330105

14. Eskazole ${ }^{\circledR}$ Tabletten. In: Rote Liste 2012. Frankfurt/Main: Rote Liste Service $\mathrm{GmbH}$; 2012. p. 387.

15. Caumes E. Efficacy of albendazole ointment on cutaneous larva migrans in 2 young children. Clin Infect Dis. 2004 Jun;38(11):1647-8. DOI: 10.1086/421026

16. Caumes E, Carriere J, Datry A, Gaxotte P, Danis M, Gentilini M. A randomized trial of ivermectin versus albendazole for the treatment of cutaneous larva migrans. Am J Trop Med Hyg. 1993 Nov;49(5):641-4.

17. Suputtamongkol Y, Kungpanichkul N, Silpasakorn S, Beeching NJ. Efficacy and safety of a single-dose veterinary preparation of ivermectin versus 7-day high-dose albendazole for chronic strongyloidiasis. Int J Antimicrob Agents. 2008 Jan;31(1):46-9. DOI: 10.1016/j.jijantimicag.2007.08.014

18. Caumes E, Datry A, Paris L, Danis M, Gentilini M, Gaxotte P. Efficacy of ivermectin in the therapy of cutaneous larva migrans. Arch Dermatol. 1992 Jul;128(7):994-5. DOI: 10.1001/archderm.1992.01680170130027

19. Louis FJ, de Quincenet G, Louis JP. Intérêt de l'ivermectine en prise unique dans le traitement du syndrome de larva migrans cutanée [Value of single-dose ivermectin in the treatment of cutaneous larva migrans syndrome]. Presse Med. 1992 Sep;21(31):1483.

20. Bouchaud O, Houzé S, Schiemann R, Durand R, Ralaimazava P, Ruggeri C, Coulaud JP. Cutaneous larva migrans in travelers: a prospective study, with assessment of therapy with ivermectin. Clin Infect Dis. 2000 Aug;31(2):493-8. DOI: 10.1086/313942

21. Vanhaecke C, Perignon A, Monsel G, Regnier S, Bricaire F, Caumes $E$. The efficacy of single dose ivermectin in the treatment of hookworm related cutaneous larva migrans varies depending on the clinical presentation. J Eur Acad Dermatol Venereol. 2013 Feb. DOI: 10.1111/jdv.12097

22. Schuster A, Lesshafft H, Reichert F, Talhari S, de Oliveira SG, Ignatius R, Feldmeier H. Hookworm-related cutaneous larva migrans in northern Brazil: resolution of clinical pathology after a single dose of ivermectin. Clin Infect Dis. 2013 Oct;57(8):11557. DOI: $10.1093 / \mathrm{cid} / \mathrm{cit} 440$

23. Veraldi S, Bottini S, Rizzitelli G, Persico MC. One-week therapy with oral albendazole in hookworm-related cutaneous larva migrans: a retrospective study on 78 patients. J Dermatolog Treat. 2012 Jun;23(3):189-91. DOI: 10.3109/09546634.2010.544707

24. Blackwell V, Vega-Lopez F. Cutaneous larva migrans: clinical features and management of 44 cases presenting in the returning traveller. Br J Dermatol. $2001 \mathrm{Sep} ; 145(3): 434-7$. DOI: 10.1046/j.1365-2133.2001.04406.x

25. Caumes E. Treatment of cutaneous larva migrans. Clin Infect Dis. 2000 May;30(5):811-4. DOI: 10.1086/313787

26. Sanguigni S, Marangi M, Teggi A, De Rosa F. Albendazole in the therapy of cutaneous larva migrans. Trans R Soc Trop Med Hyg. 1990 Nov-Dec;84(6):831.

27. Sunderkötter $\mathrm{C}$, von Stebut E, Schöfer H, Mempel M, Reinel D, Wolf G, Meyer V, Nast A, Burchard GD. S1 guideline diagnosis and therapy of cutaneous larva migrans (creeping disease). J Dtsch Dermatol Ges. 2014 Jan;12(1):86-91. DOI: $10.1111 /$ ddg. 12250 


\section{Korrespondenzadresse:}

Prof. Dr. Cord Sunderkötter, MD

Klinik für Hautkrankheiten - allgemeine Dermatologie und Venerologie, Universitätsklinikum Münster (UKM), von-Esmarch-Straße 58, 48149 Münster, Deutschland, Tel.: +49 (0)251 83 52940, Fax: +49 (0)251 8357488 Cord.Sunderkoetter@ukmuenster.de

\section{Bitte zitieren als}

Sunderkötter $C$, von Stebut E, Schöfer H, Mempel M, Reinel D, Wolf G, Meyer V, Nast A, Burchard GD. S1 Leitlinie zur Diagnostik und Therapie der kutanen Larva migrans (Creeping Disease). GMS Infect Dis.

2014;2:Doc03.

DOI: 10.3205/id000011, URN: urn:nbn:de:0183-id0000115

\section{Artikel online frei zugänglich unter}

http://www.egms.de/en/journals/id/2014-2/id000011.shtml

Veröffentlicht: 16.04.2014

\section{Copyright}

(C)2014 Sunderkötter et al. Dieser Artikel ist ein Open Access-Artikel und steht unter den Creative Commons Lizenzbedingungen (http://creativecommons.org/licenses/by-nc-nd/3.0/deed.de). Er darf vervielfältigt, verbreitet und öffentlich zugänglich gemacht werden, vorausgesetzt dass Autor und Quelle genannt werden. 


\section{S1 guideline diagnosis and therapy of cutaneous larva migrans (creeping disease)}

\begin{abstract}
Cutaneous larva migrans is a skin infection with a typical clinical appearance caused by active penetration of nematode larvae and their subsequent epidermal migration. The typical clinical appearance is caused by hookworm larvae, usually Ancylostoma braziliense but sometimes other canine or feline types of hookworm.

This guideline aims to enhance patient care by optimizing the diagnosis and treatment of infections due to creeping disease (cutaneous larva migrans) and to raise awareness among doctors of current treatment options.
\end{abstract}

\author{
Cord Sunderkötter ${ }^{1}$ \\ Esther von Stebut ${ }^{2}$ \\ Helmut Schöfer ${ }^{3}$ \\ Martin Mempel $^{4}$ \\ Dieter Reinel ${ }^{5}$ \\ Gerd Wolf ${ }^{6}$ \\ Volker Meyer $^{1}$ \\ Alexander $\mathrm{Nast}^{7}$
}

Gerd-Dieter Burchard $^{8,9}$

1 Department of Dermatology and Venereology, Münster University Hospital, Münster, Germany

2 Department of Dermatology, University Hospital of Mainz, Germany

3 Department of Dermatology, Venereology and Allergology, Johann Wolfgang Goethe University Hospital, Frankfurt, Germany

4 Elmshorn, Germany

5 Hamburg, Germany

6 Grafschaft-Ringen, Germany

7 Division of Evidence based Medicine (dEBM), Department of Dermatology, Venereology and Allergology, Charité - Universitätsmedizin Berlin, Germany

8 Division of Tropical Medicine and Infectious Diseases, University Medical Center Hamburg-Eppendorf, Hamburg, Germany

9 ifi - Institute for Interdisziplinary Medicine, Hamburg, Germany 


\section{Guideline objectives}

These guidelines aim to enhance patient care by optimizing the diagnosis and treatment of infections due to creeping disease (cutaneous larva migrans) and to raise awareness among doctors of current treatment options.

\section{Methods}

S1 guideline, non-systematic literature search, consensus process using a circular letter

\section{Introduction}

Cutaneous larva migrans is a skin infection with a typical clinical appearance caused by active penetration of nematode larvae and their subsequent epidermal migration. The typical clinical appearance is caused by hookworm larvae, usually Ancylostoma braziliense but sometimes other canine or feline types of hookworm. These hookworm larvae that are pathogenic to animals cannot reproduce in human beings.

Cutaneous larva migrans should be distinguished from larva currens. The latter is caused by Strongyloides stercoralis larvae and usually occurs on the trunk or buttocks. Migration is less irregular and produces broad, less clearly demarcated tracks on the extremities (progressing up to $5 \mathrm{~cm}$ per hour, hence the name larva currens) and usually disappears rapidly. This guideline does not address larva currens. Cutaneous larva migrans should also be distinguished from the symptom "creeping eruption" which is a migrating subcutaneous swelling with redness and urticaria of the overlying skin. It occurs, for instance, in gnathostomiasis, cutaneous paragonimiasis (Paragonimus infection), and fascioliasis (liver fluke infection by Fasciola hepatica). Similar migratory symptoms may also occur in myiasis (migratory myiasis) caused by fly larva.

\section{Pathogens}

In cutaneous larva migrans due to nematode larvae which are pathogenic to animals (Ancylostoma braziliense, Ancylostoma caninum, or Uncinaria stenocephala), the infection remains limited to the skin. The larvae penetrate the intact epidermis (e.g., feet, buttocks, or other exposed areas), presumably through the hair follicles or sweat glands, before entering the epidermis where they produce characteristic winding tracks. They do not penetrate the basement membrane. The speed of migration depends on the species, but it is generally dose not exceed one centimeter per day. The pruritic lesions are an expression of an immune response to the larvae and their products. These parasites attack mainly dogs and cats. Human infection is accidental, and the larvae die after only a few weeks.

\section{Epidemiology}

Cutaneous larva migrans is widespread in warm, sandy places (especially beaches) in South America, Africa, the Caribbean, Southeast Asia, the southern USA, and in Mediterranean regions [1].

Prevalence is high in regions with a warm, humid climate where people walk around barefoot and come into contact with animal excrement. In Germany, the disease is usually seen in patients who have vacationed in areas with beaches where there are infected, roaming animals. Analyses by international observation networks on disease in travelers and migrants have shown that cutaneous larva migrans is one of the most commonly imported skin diseases. A worldwide study found that cutaneous larva migrans makes up $10 \%$ of dermatological diagnoses in returning travelers [2]. Analyses from individual centers also report that cutaneous larva migrans is one of the most common diagnoses (e.g., in France [3] and Spain [4]).

\section{Clinical appearance of larva migrans}

A few days after contact with infected soil, a papule appears, then a winding tract develops and there is a local inflammatory reaction (Figure 1). The incubation period can last days, weeks, or months [5]. Simultaneous infestation with multiple nematodes is also possible (Figure 2). The tracts may be filled with serous fluid. Blisters may develop due to the inflammatory reaction. Pruritus is a typical symptom. Itching may begin before lesions appear, and it worsens significantly over the course of disease [6]. The cutaneous form of disease is self-limiting, because the larvae cannot survive in humans. Occasionally, lesions may persist for several months without histological detection of larvae; the cause is uncertain and may be due to persistence of larvae or a prolonged immune response to the larval debris [7]. A secondary bacterial infection (such as erysipelas) can develop from the initial entry site or because of excoriations. This complication is especially common in endemic regions.

\section{Prophylaxis}

Patients should wear shoes (or sandals) when walking on potentially contaminated beaches and soil. Bathers should not lie on the sand or even on towels, but rather should use a mattress or chair. Beachgoers should try to visit areas of the beach where the sand is regularly washed away by the tides. If possible, vacationers should avoid beaches where there are cats or dogs. 


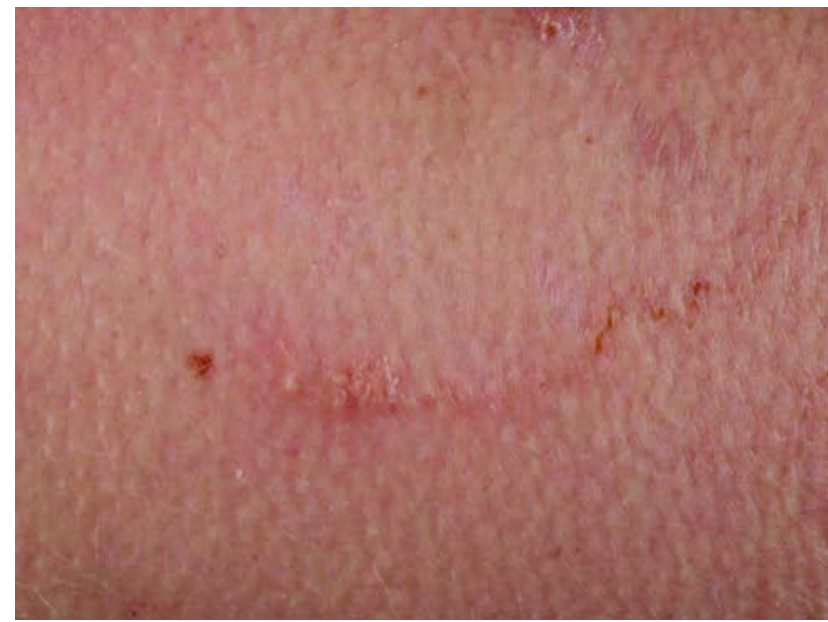

Figure 1: Serpiginous track of cutaneous larva migrans with only moderate inflammatory reaction

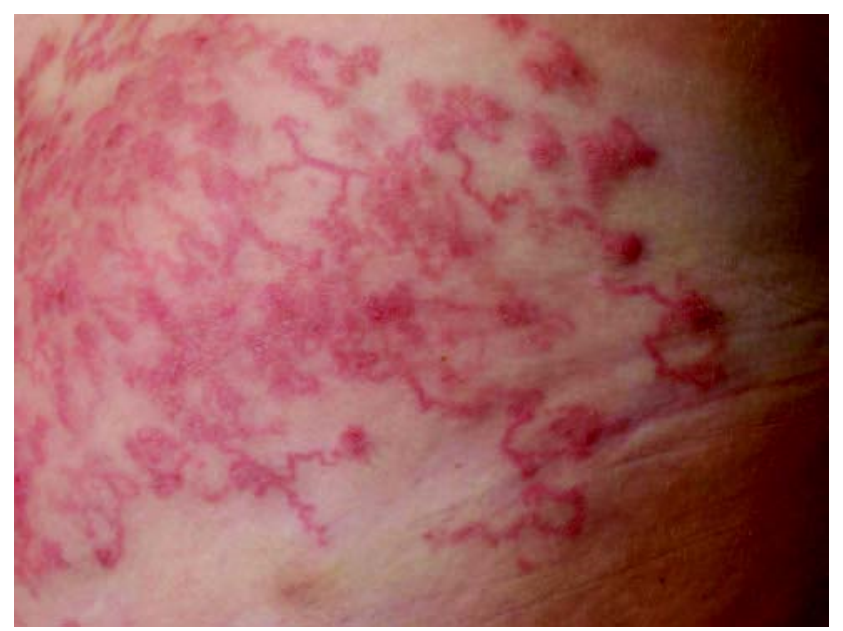

Figure 2: Infestation with several nematodes and the accompanying inflammatory reaction

\section{Diagnosis/differential diagnosis}

The serpiginous exanthems are pathognomonic. Suspicion is confirmed by a history of travel and exposure.

In infestation with Strongyloides stercoralis, the lesions usually begin in the perineal region and spread in wide, and less sharply demarcated, tracks along the extremities, moving much more rapidly (up to $5 \mathrm{~cm}$ per hour, hence the name larva currens).

\section{Indication for treatment}

Infection with cutaneous larva migrans is self-limited and generally ends after 1-3 months with the death and resorption of the larvae. Treatment is mainly required because of the severe, lasting pruritus, the psychological burden of parasite infection, and the risk of a possible superinfection (such as after excoriation due to scratching).

\section{Therapy}

In Germany and other countries, for years the recommended treatment was topical thiabendazole in a lipophilic base [8]. Although there were virtually no studies, it was believed to have a broad therapeutic spectrum. Thiabendazole has not been approved for use in Germany since 1988 , nor is it available in its pure form as a pharmaceutical grade drug.

After consulting the literature, and based on our own experience, we suggest the following treatment:

\section{Adults and adolescents:}

1. Ivermectin $1 \times 200 \mu \mathrm{g} / \mathrm{kg}$ of body weight (Stromectol ${ }^{\circledR}$ $3 \mathrm{mg}$, Mectizan ${ }^{\circledR} 3 \mathrm{mg}$, Ivermec ${ }^{\circledR} 6 \mathrm{mg}$, Revectina ${ }^{\circledR}$ $6 \mathrm{mg}$ tablet), oral administration. Patients should avoid eating anything for two hours before or after taking the medication. In general, a single dose is sufficient; if there is no improvement after 10 days, a second dose may be given [9]. In patients with severe disease, if ivermectin is unavailable, ineffective, or not well-tolerated, systemic albendazole may be an alternative.

2. Oral albendazole $800 \mathrm{mg} /$ daily for 3 days (e.g., Eskazole $^{\circledR} 400 \mathrm{mg}$ tablets). In young people weighing less than $60 \mathrm{~kg}$ who are more than 6 years old: $15 \mathrm{mg} / \mathrm{kg}$ of body weight up to a maximum of $800 \mathrm{mg} /$ daily). If the drug is not tolerated (gastrointestinal complaints), reduce dosage to $400 \mathrm{mg} /$ daily for 5 days.

Take with meals in 2 single doses in the morning and evening.

3. Topical albendazole $10 \%$ in a lipophilic base (e.g., albendazole 1,200 $\mathrm{mg}$ which corresponds to 3 tablets of $400 \mathrm{mg}$ in white Vaseline ad 12.0) three times daily for 7-10 days. Apply generously. Used mainly in children weighing less than $15 \mathrm{~kg}$ (no ivermectin) and children under age 6 (inadequate experience with albendazole). Also used in patients with discrete lesions (e.g., a single lesion or mild itching) or those who refuse systemic therapy. (See also comments below on treatment with topical albendazole).

To reduce inflammation and itching, we also recommend brief topical treatment (1-5 days) with a corticosteroid cream (expert opinion) and, if necessary, systemic antihistamines to stop the itching.

\section{Pregnancy and nursing:}

Ivermectin and albendazole must not be used by pregnant women. We can only recommend the symptomatic treatment described above; to alleviate inflammation and itching, topical treatment consisting of a corticosteroid cream may be given. If necessary, systemic antihistamines, which are approved for use in pregnant women, may be administered as well to stop the itching.

Effective treatment significantly reduces itching in 3 days and dermatitis after 7 days. 
The following treatments are not recommended:

1. Topical mebendazole. Reasons: a) Compared to albendazole, it appears to be less effective in larva migrans (and in general against hookworms) [10], [11]; b) intestinal resorption is inadequate, and thus at normal dosages, it is effective against intestinal nematodes, but not against nematodes in tissue; c) we found no publications on systemic administration of mebendazole to treat larva migrans.

2. Cryotherapy (although sometimes advised), given that the location of the larvae (usually $1-2 \mathrm{~cm}$ around the visible end of the track) is not precisely known [12] and their low sensitivity to cold skin temperatures (>5 minute survival at $-21^{\circ} \mathrm{C}$ ).

\section{Comments on ivermectin}

Ivermectin is approved for use in Germany only in veterinary medicine and is sold under the name Eraquell Tabs ${ }^{\circledR}$ $20 \mathrm{mg}$. In France, Belgium, and the United States, the drug is approved for use in humans for the treatment of the related visceral form of strongyloidiasis due to Strongyloides stercoralis.

The tablets may be obtained from foreign pharmacies as Stromectol $^{\circledR} 3 \mathrm{mg}$, Ivermec ${ }^{\circledR} 6 \mathrm{mg}$, or Revectina ${ }^{\circledR} 6 \mathrm{mg}$ (N1). The patient should be informed that ivermectin is not available in Germany nor officially recommended in other countries as larva migrans treatment. The discussion and the risk/benefit assessment of off-label use should be documented in the patient's file.

Its use is contraindicated in children weighing less than $15 \mathrm{~kg}$ and in pregnant women. Adverse effects limiting its use are transitory and have rarely been reported, despite having been used millions of times to treat onchocercosis [13].

\section{Comments on systemic albendazole}

Systemic albendazole is currently only available in packages containing 60 tablets (N3) which cost $590.38 €$ (imports: 549.09-556.09 €/Prices from: 05/2013, as Eskazole $\left.^{\circledR}[14]\right)$. Smaller sizes would be desirable, given that the incidence of larva migrans is more than negligible, and thus small amounts are needed rather frequently.

Pharmacies that provide Eskazole ${ }^{\circledR}$ have the right to charge for the entire package, even if only 6 tablets are used in accordance with the legally binding. Hospital pharmacies may only charge for the amount actually used. Theoretically, one could apply this to pharmacies as well, but such a change conflicts with the "pharmacy tax". Albendazole is approved as an anthelminthic in Germany, including for attempted treatment of Strongyloides stercoralis (see above) infections, but not for treatment of larva migrans, so that treatment of the latter is off-label. Patients should be informed of this, and the discussion as well as the risk/benefit assessment should be documented in the patient's file.

\section{Comments on topical albendazole}

The suggested formulation is based on a case report [15] albendazole $1.2 \mathrm{~g}$, white Vaseline ad $12 \mathrm{~g}$.

One co-author uses albendazole 10.0, paraffin oil 10.0, white petrolatum ad 100.0.

If Eskazole ${ }^{\circledR}$ is prescribed, the pharmacy has the right to charge full price, even if only 3 tablets are needed (see above).

Albendazole has also been offered since 2013 to pharmacies in its pure form (Fagron, Barsbüttel, Germany). This would reduce the cost of producing a topical treatment. In our own experience, there have been problems obtaining the product, apparently due to impure substances from different providers. In addition, after delivery of a pure substance with a certificate of analysis, before creating the albendazole formulation, the pharmacy must also conduct an analysis for identification of the substance using infrared spectroscopy and thin-layer chromatography. Yet, only a few pharmacies can perform infrared spectroscopy.

Thus physicians should first find out (a) which pharmacy can perform the identification procedure, and (b) whether it can order albendazole. The pharmacy may need to order, and pay for more (if there is a minimum amount) than an individual prescription requires.

If these requirements are met, however, the cost is much lower for the patient than topical Eskazole ${ }^{\circledR}$. We therefore recommend for centers which often treat patients with larva migrans that they contact a pharmacy, discuss the situation, and establish cooperation with that pharmacy to avoid having to start from scratch each time.

\section{Assessment of effectiveness}

There are only a few comparative prospective randomized studies on effectiveness of the drug. A single administration of ivermectin ( $n=10$ patients) was shown in one comparative randomized study to be more effective than $400 \mathrm{mg}$ albendazole ( $n=11$ patients) [16]. Compared to albendazole, ivermectin was shown in a prospective study on strongyloidiasis (i.e., a disease caused by a related type of worm) that a single dose was more effective than a 7-day regimen of $800 \mathrm{mg}$ albendazole [17].

In addition to comparative studies, there are a number of partly retrospective analyses on individual medications. Ivermectin: Two smaller studies from the 1990s showed a single dose to be effective in patients in Cameroon and in French tourists [18], [19]. These findings were later confirmed by larger studies [9]. In a study done in Paris, 62 Europeans were treated with ivermectin; after receiving a single dose, the healing rate was $77 \%$; it was $97 \%$ in patients who received one or two additional doses [20]. Another study from France reported healing in 59 out of 62 patients who were treated with ivermectin [21]. A study from an endemic region in Brazil reported that all 92 patients, some of whom had severe disease, had a significant response 2-4 weeks after being given a single dose of $200 \mu \mathrm{g} / \mathrm{kg}$ of body weight [22]. 
Albendazole: A retrospective study that included 78 patients with cutaneous larva migrans found that, after being given $1 \times 400 \mathrm{mg} /$ daily of oral albendazole, all patients healed after 7 days. Two patients reported nausea or increased pruritus related to the drug [23]. Other published case series and retrospective analyses have reported different dosages and treatment durations. Three days of $800 \mathrm{mg} /$ daily reportedly led to healing in $100 \%$ of patients; $400 \mathrm{mg} /$ daily led a few treatment failures [24], [25]. At a dosage of $800 \mathrm{mg} /$ daily, the rate of side effects is higher (usually gastrointestinal complaints) than with ivermectin [26].

For topical albendazole therapy, we found a case report [15] and a recommendation in the framework of a review [11]. In one case report, topical albendazole was given to 2 children, and led to recurrence-free healing 1 week after treatment [15]. Other than this case report, there are insufficient data on skin penetration, effectiveness, and tolerability. The authors of the present guidelines have also successfully treated individual patients with topical application of $10 \%$ albendazole. The recommendation to generously apply albendazole $10 \%$ in a lipophilic base 3 times daily for $7-10$ days arose analogous to the thiabendazole-based formulation that was formerly used and according to the published case report. The size of the area of application is based on the knowledge that the larvae often migrate several centimeters farther than the inflammation.

Corticosteroids: There is no direct evidence of the effectiveness of accompanying local treatment with corticosteroids to alleviate the inflammation and itching in patients with larva migrans.

\section{Notes}

\section{Validity}

The present guidelines are valid until April 2017. The need for an update will be decided on by the authors. Date created: May 2013.

\section{ICD Code (ICD 10)}

B76.9

\section{AWMF registry number}

$013 / 087$

\section{Conflicts of interest}

See Attachment 1.

\section{Financing}

The guidelines were financed solely by the authors. No funding was received and there was no outside influence on the contents of the guidelines.

\section{Permissions}

Authorized by Deutsche Dermatologische Gesellschaft (DDG).

Authorized for electronic publication: AWMF online. Publication in GMS ID has been permitted by John Wiley \& Sons Ltd in the context of the inderdisciplinary compilation of these guidelines in cooperation with the PEG (originally published in Journal der Deutschen Dermatologischen Gesellschaft 2014 [27]).

\section{Cooperating professional associations and their representatives}

- The German Society of Dermatology (DDG)/Working Group on Dermatological Infectious Diseases and Tropical Dermatology (ADI-TD): E. von Stebut-Borschitz, H. Schöfer, M. Mempel, D. Reinel, V. Meyer, A. Nast, C. Sunderkötter

- German Society for Tropical Medicine and International Health e.V. (DTG): G.-D. Burchard, D. Reinel

- Society for Dermatology in the Tropics: D. Reinel

- Paul-Ehrlich-Gesellschaft: C. Sunderkötter

\section{Attachments}

Available from

http://www.egms.de/en/journals/id/2014-2/id000011.shtml

1. Anhang1_id000011.pdf (122 KB)

Conflicts of interest (in German)

\section{References}

1. Galanti B, Fusco FM, Nardiello S. Outbreak of cutaneous larva migrans in Naples, southern Italy. Trans R Soc Trop Med Hyg. 2002 Sep-Oct;96(5):491-2. DOI: 10.1016/S00359203(02)90415-3

2. Lederman ER, Weld LH, Elyazar IR, von Sonnenburg F, Loutan L, Schwartz E, Keystone JS; GeoSentinel Surveillance Network. Dermatologic conditions of the ill returned traveler: an analysis from the GeoSentinel Surveillance Network. Int J Infect Dis. 2008 Nov;12(6):593-602. DOI: 10.1016/j.ijid.2007.12.008

3. Caumes E, Carrière J, Guermonprez G, Bricaire F, Danis M, Gentilini M. Dermatoses associated with travel to tropical countries: a prospective study of the diagnosis and management of 269 patients presenting to a tropical disease unit. Clin Infect Dis. 1995 Mar;20(3):542-8. DOI: 10.1093/clinids/20.3.542

4. Ramírez-Olivencia G, Bru Gorraiz FJ, Rivas González P, Lago Núñez M, Herrero Mendoza MD, Puente Puente S. Patologia dermatologica y medicina tropical. Resultados de un estudio prospectivo (2004-2007)[Skin diseases and tropical medicine. Results from a prospective study (2004-2007)]. Rev Clin Esp. 2009 Dec;209(11):527-35. DOI: 10.1016/S00142565(09)73058-9

5. Siriez JY, Angoulvant F, Buffet P, Cleophax C, Bourrat E. Individual variability of the cutaneous larva migrans (CLM) incubation period. Pediatr Dermatol. 2010 Mar-Apr;27(2):211-2. DOI: 10.1111/j.1525-1470.2010.01107.x

6. Feldmeier H, Schuster A. Mini review: Hookworm-related cutaneous larva migrans. Eur J Clin Microbiol Infect Dis. 2012 Jun;31(6):915-8. DOI: 10.1007/s10096-011-1404-x 
7. Veraldi S, Persico MC, Francia C, Schianchi R. Chronic hookworm related cutaneous larva migrans. Int J Infect Dis. 2013 Apr;17(4):e277-9. DOI: 10.1016/j.ijid.2012.11.002

8. Hochedez P, Caumes E. Hookworm-related cutaneous larva migrans. J Travel Med. 2007 Sep-Oct;14(5):326-33. DOI: 10.1111/j.1708-8305.2007.00148.x

9. Van den Enden E, Stevens A, Van Gompel A. Treatment of cutaneous larva migrans. N Engl J Med. 1998 Oct 22;339(17):1246-7. DOI: 10.1056/NEJM199810223391714

10. Steinmann P, Utzinger J, Du ZW, Jiang JY, Chen JX, Hattendorf J, Zhou $\mathrm{H}$, Zhou XN. Efficacy of single-dose and triple-dose albendazole and mebendazole against soil-transmitted helminths and Taenia spp.: a randomized controlled trial. PLOS ONE. 2011;6(9):e25003. DOI: 10.1371/journal.pone.0025003

11. Wolf $\mathrm{P}$, Ochsendorf FR, Milbradt R. Aktuelle Therapiemöglichkeiten bei Larva migrans cutanea [Current therapeutic possibilities in cutaneous larva migrans]. Hautarzt. 1993 Jul;44(7):462-5.

12. Albanese G, Venturi C, Galbiati G. Treatment of larva migrans cutanea (creeping eruption): a comparison between albendazole and traditional therapy. Int J Dermatol. 2001 Jan;40(1):67-71. DOI: 10.1046/j.1365-4362.2001.01103.x

13. Meinking TL, Taplin D, Hermida JL, Pardo R, Kerdel FA. The treatment of scabies with ivermectin. N Engl J Med. 1995 Jul;333(1):26-30. DOI: 10.1056/NEJM199507063330105

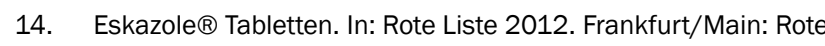
Liste Service $\mathrm{GmbH}$; 2012. p. 387.

15. Caumes E. Efficacy of albendazole ointment on cutaneous larva migrans in 2 young children. Clin Infect Dis. 2004 Jun;38(11):1647-8. DOI: 10.1086/421026

16. Caumes E, Carriere J, Datry A, Gaxotte P, Danis M, Gentilini M. A randomized trial of ivermectin versus albendazole for the treatment of cutaneous larva migrans. Am J Trop Med Hyg. 1993 Nov;49(5):641-4.

17. Suputtamongkol Y, Kungpanichkul N, Silpasakorn S, Beeching $\mathrm{NJ}$. Efficacy and safety of a single-dose veterinary preparation of ivermectin versus 7-day high-dose albendazole for chronic strongyloidiasis. Int J Antimicrob Agents. 2008 Jan;31(1):46-9. DOI: 10.1016/j.ijantimicag.2007.08.014

18. Caumes E, Datry A, Paris L, Danis M, Gentilini M, Gaxotte P. Efficacy of ivermectin in the therapy of cutaneous larva migrans. Arch Dermatol. 1992 Jul;128(7):994-5. DOI: 10.1001/archderm.1992.01680170130027

19. Louis FJ, de Quincenet G, Louis JP. Intérêt de l'ivermectine en prise unique dans le traitement du syndrome de larva migrans cutanée [Value of single-dose ivermectin in the treatment of cutaneous larva migrans syndrome]. Presse Med. 1992 Sep;21(31):1483.

20. Bouchaud O, Houzé S, Schiemann R, Durand R, Ralaimazava P, Ruggeri C, Coulaud JP. Cutaneous larva migrans in travelers: a prospective study, with assessment of therapy with ivermectin. Clin Infect Dis. 2000 Aug;31(2):493-8. DOI: 10.1086/313942

21. Vanhaecke C, Perignon A, Monsel G, Regnier S, Bricaire F, Caumes $E$. The efficacy of single dose ivermectin in the treatment of hookworm related cutaneous larva migrans varies depending on the clinical presentation. J Eur Acad Dermatol Venereol. 2013 Feb. DOI: $10.1111 / \mathrm{jdv} .12097$
22. Schuster A, Lesshafft H, Reichert F, Talhari S, de Oliveira SG Ignatius R, Feldmeier $\mathrm{H}$. Hookworm-related cutaneous larva migrans in northern Brazil: resolution of clinical pathology after a single dose of ivermectin. Clin Infect Dis. 2013 Oct;57(8):11557. DOI: $10.1093 / \mathrm{cid} / \mathrm{cit} 440$

23. Veraldi S, Bottini S, Rizzitelli G, Persico MC. One-week therapy with oral albendazole in hookworm-related cutaneous larva migrans: a retrospective study on 78 patients. J Dermatolog Treat. 2012 Jun;23(3):189-91. DOI: 10.3109/09546634.2010.544707

24. Blackwell V, Vega-Lopez F. Cutaneous larva migrans: clinical features and management of 44 cases presenting in the returning traveller. Br J Dermatol. 2001 Sep;145(3):434-7. DOI: 10.1046/j.1365-2133.2001.04406.x

25. Caumes E. Treatment of cutaneous larva migrans. Clin Infect Dis. 2000 May;30(5):811-4. DOI: 10.1086/313787

26. Sanguigni S, Marangi M, Teggi A, De Rosa F. Albendazole in the therapy of cutaneous larva migrans. Trans R Soc Trop Med Hyg. 1990 Nov-Dec;84(6):831.

27. Sunderkötter C, von Stebut E, Schöfer H, Mempel M, Reinel D, Wolf G, Meyer V, Nast A, Burchard GD. S1 guideline diagnosis and therapy of cutaneous larva migrans (creeping disease). J Dtsch Dermatol Ges. 2014 Jan;12(1):86-91. DOI: $10.1111 /$ ddg. 12250

\section{Corresponding author:}

Prof. Dr. Cord Sunderkötter, MD

Department of Dermatology and Venereology, Münster University Hospital, von-Esmarch-Straße 58, 48149

Münster, Germany, Phone: +49 (0)251 83 52940, Fax: +49 (0)251 8357488

\section{Cord.Sunderkoetter@ukmuenster.de}

\section{Please cite as}

Sunderkötter $C$, von Stebut E, Schöfer H, Mempel M, Reinel D, Wolf G, Meyer V, Nast A, Burchard GD. S1 Leitlinie zur Diagnostik und Therapie der kutanen Larva migrans (Creeping Disease). GMS Infect Dis. 2014;2:Doc03.

DOI: 10.3205/id000011, URN: urn:nbn:de:0183-id0000115

\section{This article is freely available from}

http://www.egms.de/en/journals/id/2014-2/id000011.shtml

\section{Published: 2014-04-16}

\section{Copyright}

(C)2014 Sunderkötter et al. This is an Open Access article distributed under the terms of the Creative Commons Attribution License (http://creativecommons.org/licenses/by-nc-nd/3.0/deed.en). You are free: to Share - to copy, distribute and transmit the work, provided the original author and source are credited. 